Arthroskopie 2015 - 28:6-7

DOI 10.1007/s00142-014-0830-z

Online publiziert: 13. Dezember 2014

c) Springer-Verlag Berlin Heidelberg 2014

C. Fink ${ }^{1} \cdot$ P. Lobenhoffer ${ }^{2}$

${ }^{1}$ Sportsclinic Austria, Innsbruck

${ }^{2}$ GO:H Gelenkchirurgie Orthopädie Hannover

\title{
Arthrose nach Meniskus- und Kreuzbandläsion
}

Liebe Leserinnen und Leser,

Meniskus- und Kreuzbandoperationen gehören heute mit Abstand zu den häufigsten arthroskopischen Eingriffen am Kniegelenk. Während der kurz- und mittelfristige Erfolg unumstritten ist, gibt es immer wieder Diskussionen, wie sich diese Eingriffe langfristig auf das Kniegelenk auswirken.

Bereits 1948 hat Fairbank [1] auf die Risiken einer Totalmeniskektomie für den Gelenkknorpel hingewiesen. Zwar mögen wir denken, dass dies Geschichte ist und wir heute mit unseren arthroskopischen Techniken weit überlegen sind, doch das stimmt leider nur zum Teil. Die Invasivität der Arthroskopie ist zwar deutlich geringer als jene der Kniearthrotomie, allerdings kann auch bereits die Resektion eines kleinen Meniskusanteils große negative Auswirkungen auf die Druckverteilung im Kniegelenk haben. Daher ist es wichtig, die biomechanischen Charakteristika jedes Risstyps und jeder Meniskusläsion zu verstehen. Gerade Wurzelläsionen oder ausgedehnte Radiärrisse können zu einem nahezu völligen Funktionsverlust des Meniskus führen, obwohl $100 \%$ der Substanz vorhanden ist. Außerdem kann auch die segmentale Resektion eines kleinen Meniskusanteils, welche an die Meniskusbasis reicht, mechanisch gesehen einer Totalresektion nahekommen (funktionelle Totalmeniskektomie).

Gerade deshalb ist die möglichst weitgehende Erhaltung der Menisken entscheidend. Die Beherrschung der unterschiedlichen Naht- und Refixationstechniken muss daher unbedingt zum Repertoire eines Kniechirurgen gehören.

Hat der Patient nach einer Meniskusteilresektion Schmerzen, gibt es heute die
Möglichkeit, Teile der Menisken durch Implantate zu ersetzten. Die notwendigen operativen Eingriffe sind technisch anspruchsvoll, und die Nachbehandlung aufwändig. Die klinischen Ergebnisse sind ermutigend, wenn auch nicht uneingeschränkt positiv. Der Weg scheint jedoch der richtige zu sein. Das Ziel, einen resezierten Meniskusanteil unmittelbar durch ein Implantat zu ersetzen und nach kurzer Nachbehandlung wieder ein funktionierendes Kniegelenk zu erreichen, bedarf jedoch noch vieler Jahre intensiver Forschung.

Fehlt der Meniskus vollständig, ist die allogene Meniskustransplantation eine interessante Option. In operationstechnischer Hinsicht hat sich in diesem Bereich auch sehr viel bewegt. Problematisch sind hier die Verfügbarkeit von passendem Meniskusgewebe sowie die in den europäischen Ländern oft hinderlichen Transplantationsrichtlinien.

Auch die Ruptur des vorderen Kreuzbands (VKB) ist mit weitreichenden Veränderungen der Kinematik des Kniegelenks verbunden. Trotz intensiver Forschung im Bereich der Anatomie und Biomechanik und trotz der Entwicklung immer neuer Operationstechniken sind wir auch in diesem Bereich der Kniechirurgie noch nicht soweit, unseren kreuzbandverletzten Patienten langfristig ein Kniegelenk ohne erhöhtes Risiko für degenerative Knorpelveränderungen versprechen zu können. Auch in neueren Metaanalysen lässt sich leider noch immer kein sicherer Hinweis dafür finden, dass die Rekonstruktion des VKB im Vergleich zur konservativen Behandlung eine eindeutige Arthroseprävention darstellt. Hier spielen natürlich das ursprüngliche Verletzungsausmaß (v. a. Zusatzverletzungen des Gelenkknorpels und der Menisken) 
sowie die sportlichen Aktivitäten der Patienten eine große Rolle.

Sind bereits degenerative Veränderungen des Gelenkknorpels vorhanden, ist im Fall einer VKB-Ruptur natürlich auch diese Problematik zu beachten. Die VKB-Rekonstruktion kann hier nur die Instabilität beheben und Zusatzeingriffe, wie z. B. Osteotomien, sind notwendig, um eine dauerhafte Funktionsverbesserung und insbesondere auch eine Verbesserung der Schmerzsituation zu gewährleisten.

Mit diesem Themenheft „Arthrose nach Meniskus und Kreuzbandläsion“" möchten wir Ihnen einen umfassenden Überblick über die aktuelle Literatur ermöglichen und Ihnen praktische und technische Tipps für Ihre tägliche Praxis geben.

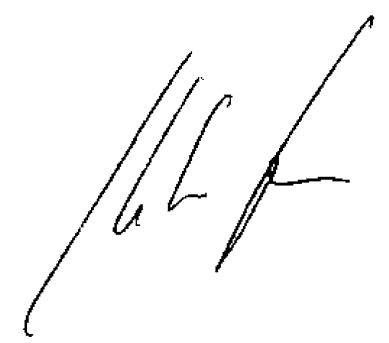

\section{Literatur \\ 1. Fairbank TJ (1948) Knee joint changes after meni- \\ scectomy. J Bone Joint Surg (Br) 30B:664-670} hungen hin: Karl Storz: Lizenzgebühren, Beratervertrag. P. Lobenhoffer gibt an, dass kein Interessenkonflikt besteht.

\section{Korrespondenzadresse}

Prof. Dr. C. Fink

Sportsclinic Austria

Olympiastr.39, 6020 Innsbruck

Österreich

christian.fink@umit.at

\section{Prof. Dr. P. Lobenhoffer}

Orthopädie Hannover

Uhlemeyerstrasse 16,

30175 Hannover

philipp.lobenhoffer

@g-o-hannover.de
GO:H Gelenkchirurgie

\section{Christian Fink}

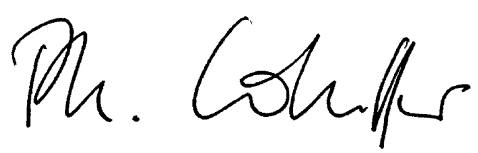

Philipp Lobenhoffer

\section{Schultergürtel, AC- und SC-Gelenke}

Bei Schulterverletzungen wird der Schultergürtel mit seinen kleinen Gelenken, wie dem AC- und dem SC-Gelenk, oft übersehen. Bereits bei kleinen Bewegungen im eigentlichen Schultergelenk, dem glenohumeralen Gelenk, finden Rotationsbewegungen im sternoklavikulären und im akromioklavikulären Gelenk statt. Anatomisch sind es deshalb ebenfalls funktionelle Kugelgelenke, auch wenn sie keine eigentliche Kugel darstellen.

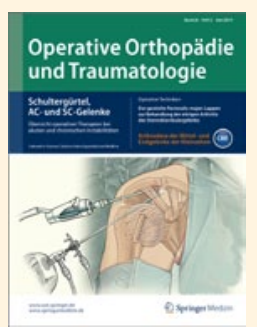

Schwierigkeiten an diesen Gelenken widmet sich Ausgabe 3/2014 der Zeitschrift „Operative Orthopädie und Traumatologie". Lesen Sie im Leitthemenheft

"Schultergürtel, AC- und SC-Gelenke" unter anderem folgende Beiträge:

- Arthroskopisch assistierte Stabilisierung bei akuter und chronischer Akromioklavikulargelenksprengung

- Offen anatomische Rekonstruktion der chronischen Akromioklavikularinstabilität - Arthroskopische Resektion des Akromioklavikulargelenks

- Die laterale Klavikulafraktur

- Arthroskopische Neurolyse und Therapie periglenoidaler Ganglien am Schultergelenk

Bestellen Sie diese Ausgabe zum Preis von 72,- EUR zzgl. Versandkosten bei Springer Customer Service Center Kundenservice Zeitschriften

Haberstr. 7

69126 Heidelberg

Tel.: +49 6221-345-4303

Fax: +49 6221-345-4229

E-Mail: leserservice@springer.com

Suchen Sie noch mehr zum Thema? Mit e.Med, dem Online-Paket von Springer Medizin, können Sie schnell und komfortabel in über 600 medizinischen Fachzeitschriften recherchieren. Weitere Infos unter springermedizin.de/eMed. 